\title{
Gadolinium Enhancement of the Aneurysm Wall in Unruptured Intracranial Aneurysms Is Associated with an Increased Risk of Aneurysm Instability: A Follow-Up Study
}

\author{
(D) M.D.I. Vergouwen, (DD. Backes, (DI.C. van der Schaaf, (D). Hendrikse, (DR. Kleinloog, (D) A. Algra, and (D) G.J.E. Rinkel
}

\begin{abstract}
BACKGROUND AND PURPOSE: Previous studies have suggested that gadolinium enhancement of the wall of unruptured intracranial aneurysms on MR imaging may reflect aneurysm wall instability. However, all previous studies were cross-sectional. In this longitudinal study, we investigated whether aneurysm wall enhancement is associated with an increased risk of aneurysm instability.
\end{abstract}

MATERIALS AND METHODS: We included all patients 18 years of age or older with $\geq 1$ unruptured aneurysm from the University Medical Center Utrecht, the Netherlands, who were included in 2 previous studies with either 3T or 7T aneurysm wall MR imaging and for whom it was decided not to treat the aneurysm but to monitor it with follow-up imaging. We investigated the risk of growth or rupture during follow-up of aneurysms with and without gadolinium enhancement of the aneurysm wall at baseline and calculated the risk difference between the 2 groups with corresponding $95 \%$ confidence intervals.

RESULTS: We included 57 patients with 65 unruptured intracranial aneurysms. After a median follow-up of 27 months (interquartile range, $20-31$ months), growth $(n=2)$ or rupture $(n=2)$ was observed in 4 of 19 aneurysms $(21 \% ; 95 \% \mathrm{Cl}, 6 \%-54 \%)$ with wall enhancement and in zero of 46 aneurysms ( $0 \% ; 95 \% \mathrm{Cl}, 0 \%-8 \%$ ) without enhancement (risk difference, $21 \% ; 95 \% \mathrm{Cl}, 3 \%-39 \%)$.

CONCLUSIONS: Gadolinium enhancement of the aneurysm wall on MR imaging is associated with an increased risk of aneurysm instability. The absence of wall enhancement makes it unlikely that the aneurysm will grow or rupture in the short term. Larger studies are needed to investigate whether aneurysm wall enhancement is an independent predictor of aneurysm instability.

ABBREVIATION: PHASES = Population, Hypertension, Age, Size of Aneurysm, Earlier Subarachnoid Hemorrhage from Another Aneurysm, and Site of Aneurysm

$\mathbf{T}$ he prevalence of unruptured intracranial aneurysms is approximately $3 \%$ in adults. ${ }^{1}$ Aneurysm rupture results in subarachnoid hemorrhage, a subtype of stroke with a poor prognosis. ${ }^{2}$ In patients with an unruptured intracranial aneurysm, the only available treatment options to prevent SAH and improve life expectancy with good quality of life are surgical clipping and endovascular treatment. Because these treatment options have a risk of complications, the risk of aneurysm rupture needs to be weighed against the risk of treatment complications. ${ }^{3}$ If the predicted risk of aneurysm rupture is small, patients are usually fol-

Received February 8, 2019; accepted after revision May 14.

From the Department of Neurology and Neurosurgery (M.D.I.V., D.B., A.A., G.J.E.R.) Brain Center Rudolf Magnus, Department of Radiology (I.C.v.d.S., J.H.), and Julius Center for Health Sciences and Primary Care, (G.J.E.R.), University Medical Center Utrecht, Utrecht University, Utrecht, the Netherlands; Department of General Practice (D.B.), Erasmus Medical Center, Rotterdam, the Netherlands; and Department of Neurology (R.K.), Academic Medical Center, Amsterdam, the Netherlands. Please address correspondence to Mervyn D.I. Vergouwen, MD, PhD, Department of Neurology and Neurosurgery, Room G3.228, Brain Center Rudolf Magnus, University Medical Center Utrecht, Utrecht University, PO Box 85 500, 3500 GA Utrecht, the Netherlands; e-mail: m.d.i.vergouwen@umcutrecht.nl

http://dx.doi.org/10.3174/ajnr.A6105 lowed across time with repeat imaging to detect possible aneurysm growth, which is a marker of aneurysm instability and a predictor of rupture.

Current risk prediction of aneurysm growth and rupture is mainly based on aneurysm size, location, and demographics. ${ }^{4,5}$ Although small aneurysms have a low risk of rupture, most episodes of SAH come from small aneurysms because small aneurysms are much more prevalent. ${ }^{6}$ Currently, most small aneurysms remain untreated because the risk of rupture is smaller than the risk of treatment complications. The clinical challenge is to identify the subgroup of small aneurysms with a high risk of rupture.

Several studies have shown that the wall of an intracranial aneurysm can enhance after administration of gadolinium using MR imaging. ${ }^{7-14}$ Enhancement of the aneurysm wall may represent aneurysm instability and therefore may be a novel biomarker to identify unstable aneurysms. However, previous studies had a cross-sectional design. Here we present the results of the first longitudinal study investigating whether gadolinium enhancement of the aneurysm wall at baseline is associated with aneurysm growth or rupture during follow-up. 


\section{MATERIALS AND METHODS}

This study was performed at the University Medical Center Utrecht, the Netherlands. Approval for this study was obtained from the local institutional review board. All patients provided written informed consent. We included all patients 18 years of age or older with $\geq 1$ unruptured aneurysm who were included in 2 previous studies with either 3T or 7T aneurysm wall MR imaging, in whom it was decided not to treat the aneurysm but to monitor it with follow-up imaging. ${ }^{12,15}$ In our center, the decision as to whether an aneurysm should be treated is based on the following parameters: the Population, Hypertension, Age, Size of Aneurysm, Earlier Subarachnoid Hemorrhage from Another Aneurysm, and Site of Aneurysm (PHASES) score for estimating the 5 -year risk of rupture, the assumed risk of complications from preventive endovascular or neurosurgical aneurysm treatment, life expectancy, and patient anxiety. ${ }^{4}$

At baseline, 3T or 7T aneurysm wall MR imaging was performed. Follow-up imaging was performed with MR angiography or CT angiography to determine potential aneurysm growth across time.

\section{Baseline Imaging}

Patients scanned on 3T MR imaging were included in the gadoLiniUM-enhanced aneurysm wall Imaging of Non-ruptured intracranial Aneurysms (LUMINA) study. ${ }^{12}$ In short, LUMINA was a cross-sectional study in which patients 18 years of age or older with $\geq 1$ unruptured intracranial aneurysm were included if the treating physician decided on follow-up imaging instead of preventive aneurysm occlusion. Exclusion criteria were impaired renal function (glomerular filtration rate of $<30 \mathrm{~mL} / \mathrm{min} / 1.73 \mathrm{~m}$ ), gadolinium contrast allergy, or pregnancy. In total, 79 patients with 89 aneurysms were recruited for the LUMINA study between October 2014 and October 2015.

Patients scanned on 7T MR imaging were recruited between February and May 2014 for a study focusing on aneurysm volume pulsation. ${ }^{15}$ In short, patients 18 years of age or older diagnosed with a saccular intradural unruptured intracranial aneurysm who were either scheduled for treatment of their aneurysm or were in follow-up to detect possible aneurysm growth were recruited through the outpatient clinic of the Department of Neurology and Neurosurgery. Exclusion criteria were the presence of contraindications for 7T MR imaging (eg, claustrophobia and metal objects such as dental implants or prostheses in or on the body), presence of an aneurysm associated with a vascular malformation other than an aneurysm (eg, an arteriovenous malformation), impaired renal function (glomerular filtration rate of $<30 \mathrm{~mL} / \mathrm{min} /$ $1.73 \mathrm{~m}$ ), and gadolinium contrast allergy.

\section{Imaging Protocol}

A 3T whole body scanner (Philips Achieva, Philips Healthcare, Best, The Netherlands) and a 7T whole-body system (Philips Healthcare, Cleveland, $\mathrm{OH}$ ) were used to acquire aneurysm wall imaging before and after administration of a single intravenous injection of a gadolinium-based contrast agent, gadobutrol (Gadovist, $0.1 \mathrm{mmol} / \mathrm{kg}$ body weight; Bayer Schering Pharma, Berlin, Germany). Imaging protocols for 3T and 7T aneurysm wall imaging were described previously. ${ }^{12,15,16}$

\section{Assessment of Baseline MR Imaging}

Two neuroradiologists (J.H. or I.C.v.d.S.) reviewed the baseline MR images blinded to patient-specific characteristics and recorded aneurysm size (maximum diameter on a $0.1-\mathrm{mm}$ scale), aneurysm location, aneurysm shape (regular or irregular shape defined as the presence of blebs, aneurysm wall protrusions, or multiple lobes), and the presence of gadolinium enhancement of the aneurysm wall (defined as present or absent).

\section{Assessment of Outcome}

The outcome measure was aneurysm instability, which was defined as aneurysm growth or rupture. The minimal follow-up time was 12 months. Aneurysm growth was defined as growth of $\geq 1 \mathrm{~mm}$ in at least 1 direction. We used radiology reports of follow-up MRA and CTA scans to determine whether aneurysm growth occurred. Radiologists who assessed the aneurysm size at follow-up imaging were blinded to the findings of aneurysm wall imaging at baseline. We checked the medical records of all patients who had aneurysm wall imaging for aneurysm rupture during follow-up. In case of death, the general practitioner was contacted to verify the cause of death.

\section{Statistical Analysis}

We investigated the risk of growth or rupture during follow-up of aneurysms with and without gadolinium enhancement of the aneurysm wall at baseline. In addition, we determined the risk difference between the 2 groups with an accompanying 95\% confidence interval.

\section{RESULTS}

Follow-up imaging was available for 55 patients with 63 asymptomatic aneurysms who had aneurysm wall MR imaging at $3 \mathrm{~T}$ and for 2 patients with 2 asymptomatic aneurysms who had aneurysm wall imaging at 7T. The last follow-up imaging was MRA for 56 aneurysms and CTA in 9 aneurysms. Reasons for not having follow-up imaging available were the following: 1) patient discharged from further follow-up because of advanced age $(n=6) ; 2)$ aneurysm treated after baseline MR imaging ( $n=2)$; or 3 ) patient awaiting follow-up imaging because of a short follow-up time $(n=13)$, death from lung cancer $(n=1)$, SAH before scheduled follow-up imaging $(n=1)$, and the patient never wanting to be treated for unruptured intracranial aneurysm $(n=1)$. None of the patients were lost during followup. Reasons for aneurysm treatment after baseline imaging were aneurysm growth compared with earlier imaging and bilobar configuration of the aneurysm, which was not visible on the earlier scan of lower quality. In both patients, the aneurysm was not symptomatic.

Patient and aneurysm characteristics of the included patients are shown in the Table. At baseline, 19 aneurysms (29\%; 95\% CI, $18 \%-46 \%$ ) had gadolinium enhancement of the wall. After a median follow-up of 27 months (interquartile range, 20-31 months), an outcome event occurred in 4 of 19 aneurysms (21\%; $95 \%$ CI, 6\%-54\%) with enhancement and in zero of 46 aneurysms ( $0 \%$; $95 \%$ CI, $0 \%-8 \%)$ without enhancement (risk difference, $21 \%$; 95\% CI, 3\%-39\%). Growth without rupture was observed in 2 aneurysms and was detected with CTA for 1 aneurysm and with MRA for 1 aneurysm (Fig 1). Aneurysm rupture occurred in 2 aneurysms (Fig 2). 


\section{DISCUSSION}

Our follow-up study showed that gadolinium enhancement of the aneurysm wall is associated with an increased risk of aneurysm instability during follow-up. One of 5 aneurysms with wall enhancement had growth or rupture, while no

\section{Patient and aneurysm characteristics at baseline ${ }^{\mathrm{a}}$}

\begin{tabular}{|c|c|c|c|}
\hline & \multicolumn{2}{|c|}{ Wall Enhancement $(n=19)$} & \multirow{2}{*}{$\begin{array}{c}\text { No Wall } \\
\text { Enhancement } \\
(n=46)\end{array}$} \\
\hline & $\begin{array}{l}\text { Unstable during } \\
\text { FU }(n=4)\end{array}$ & $\begin{array}{l}\text { Stable during } \\
\text { FU }(n=15)\end{array}$ & \\
\hline Median age (IQR) (yr) & $50(47-53)$ & $60(55-68)$ & $55(52-61)$ \\
\hline Female sex & $4(100)$ & $11(73)$ & $37(80)$ \\
\hline Hypertension & $2(50)$ & $9(60)$ & $21(46)$ \\
\hline \multicolumn{4}{|l|}{ Smoking status } \\
\hline Current smoker & $2(50)$ & $3(20)$ & $14(30)$ \\
\hline Previous smoker & $1(25)$ & $10(67)$ & $25(54)$ \\
\hline Never smoked & $1(25)$ & $2(13)$ & $7(15)^{\prime}$ \\
\hline Previous SAH & - & $3(20)$ & $11(24)$ \\
\hline Family history of intracranial aneurysms & $1(25)$ & - & $10(22)$ \\
\hline \multicolumn{4}{|l|}{ Aneurysm location } \\
\hline ICA (excluding PcomA) & - & $1(7)$ & $16(35)$ \\
\hline PcomA & - & $1(7)$ & $3(7)^{\prime}$ \\
\hline ACA/AcomA/pericallosal artery & - & $1(7)$ & $9(20)$ \\
\hline MCA & $4(100)$ & $11(73)$ & $12(26)$ \\
\hline Basilar artery & - & $1(7)^{\prime}$ & $2(4)^{\prime}$ \\
\hline Other posterior circulation & - & - & $4(9)$ \\
\hline \multicolumn{4}{|l|}{ Aneurysm size (mm) } \\
\hline $1.0-2.9$ & - & $1(7)$ & $16(35)$ \\
\hline $3.0-4.9$ & - & $3(20)$ & $16(35)$ \\
\hline $5.0-6.9$ & $3(75)$ & $6(40)$ & $11(24)$ \\
\hline$\geq 7.0$ & $1(25)$ & $5(33)$ & $3(7)$ \\
\hline Irregular shape & - & $6(40)$ & $9(20)$ \\
\hline Median follow-up duration (IQR) (mo) & $15(12-28)$ & $27(19-31)$ & $27(24-32)$ \\
\hline
\end{tabular}

Note:-PcomA indicates posterior communicating artery; AcomA, anterior communicating artery; ACA, anterior cerebral artery; FU, follow-up; IQR, interquartile range; MCA, middle cerebral artery; SAH, subarachnoid hemorrhage; -, no patients.

${ }^{a}$ Data are aneurysm-based instead of patient-based and are presented as number (\%) unless stated otherwise. growth or rupture was observed in aneurysms without wall enhancement.

The current study differs from previous studies. This is the first longitudinal study to investigate whether gadolinium enhancement of the aneurysm wall is associated with aneurysm instability during follow-up, while previous studies had a cross-sectional design..$^{7-11,14}$ Those studies often compared the proportion of patients with gadolinium enhancement of the aneurysm wall in stable and unstable (mostly ruptured) intracranial aneurysms..$^{7-11,14}$ In a small study of 5 patients with subarachnoid hemorrhage of whom 3 had multiple intracranial aneurysms, gadolinium enhancement of the aneurysm wall was present in all ruptured but not in the unruptured aneurysms. ${ }^{7}$ The second study found aneurysm wall enhancement in $29 \%$ of the 77 unruptured aneurysms, and in $87 \%$ of 31 unstable aneurysms, which were defined as a recently ruptured aneurysm, symptomatic aneurysm, or an aneurysm undergoing morphologic change. ${ }^{8}$ A third study found aneurysm wall enhancement in $18 \%$ of 83 unruptured intracranial aneurysms and in $98 \%$ of 61 ruptured intracranial aneurysms. ${ }^{9}$ A fourth study found aneurysm wall enhancement in $11 \%$ of the 18 unruptured, stable aneurysms and in all
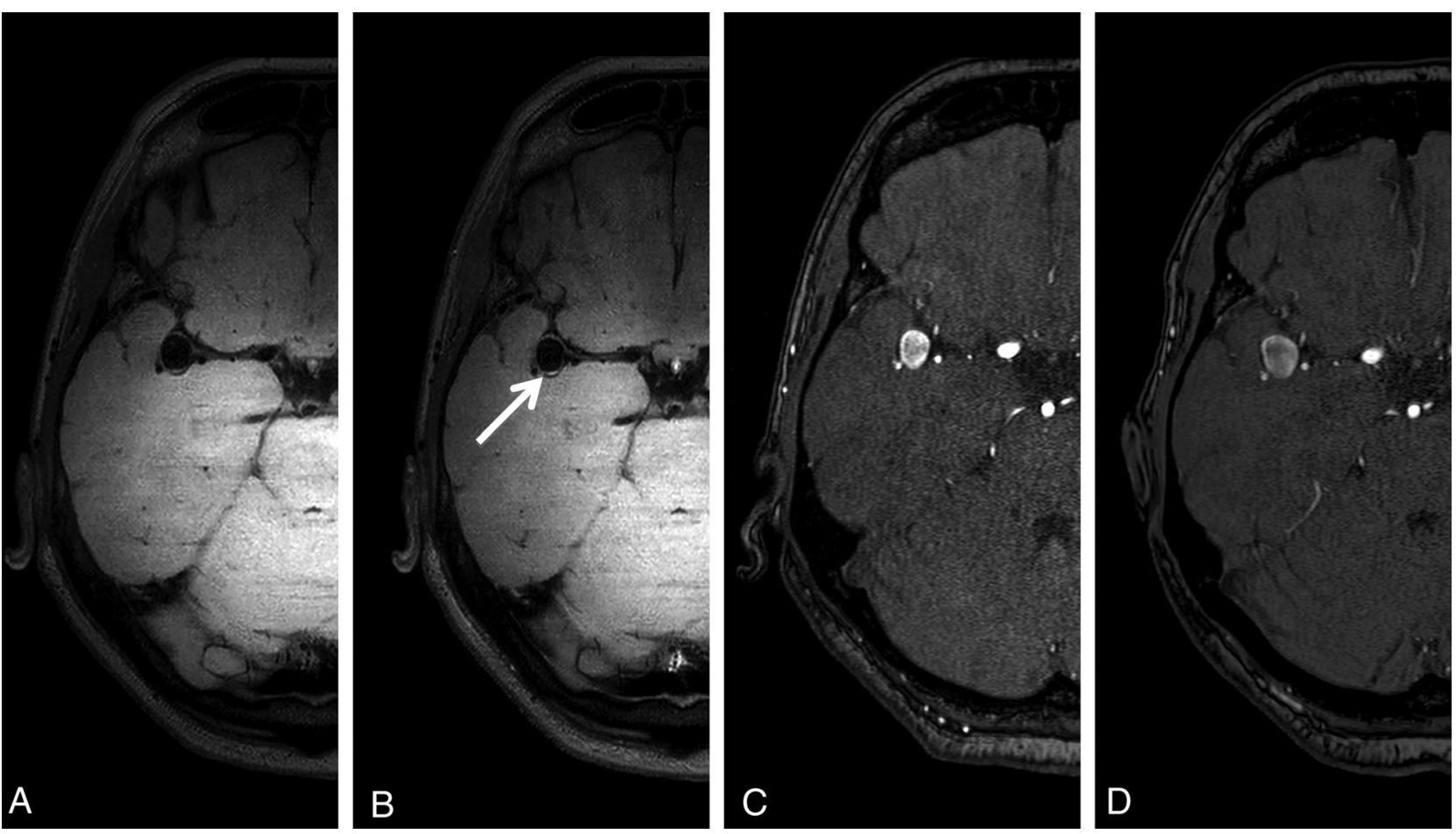

FIG 1. Aneurysm wall imaging of a patient with aneurysm growth at follow-up. A 63-year-old woman with a right middle cerebral artery aneurysm. A, Aneurysm wall imaging without gadolinium. B. Aneurysm wall imaging after administration of gadolinium shows focal posterior enhancement (arrow). C, TOF-MRA at baseline shows a 9-mm aneurysm. D, TOF-MRA after 12 months of follow-up shows a 12-mm aneurysm. 

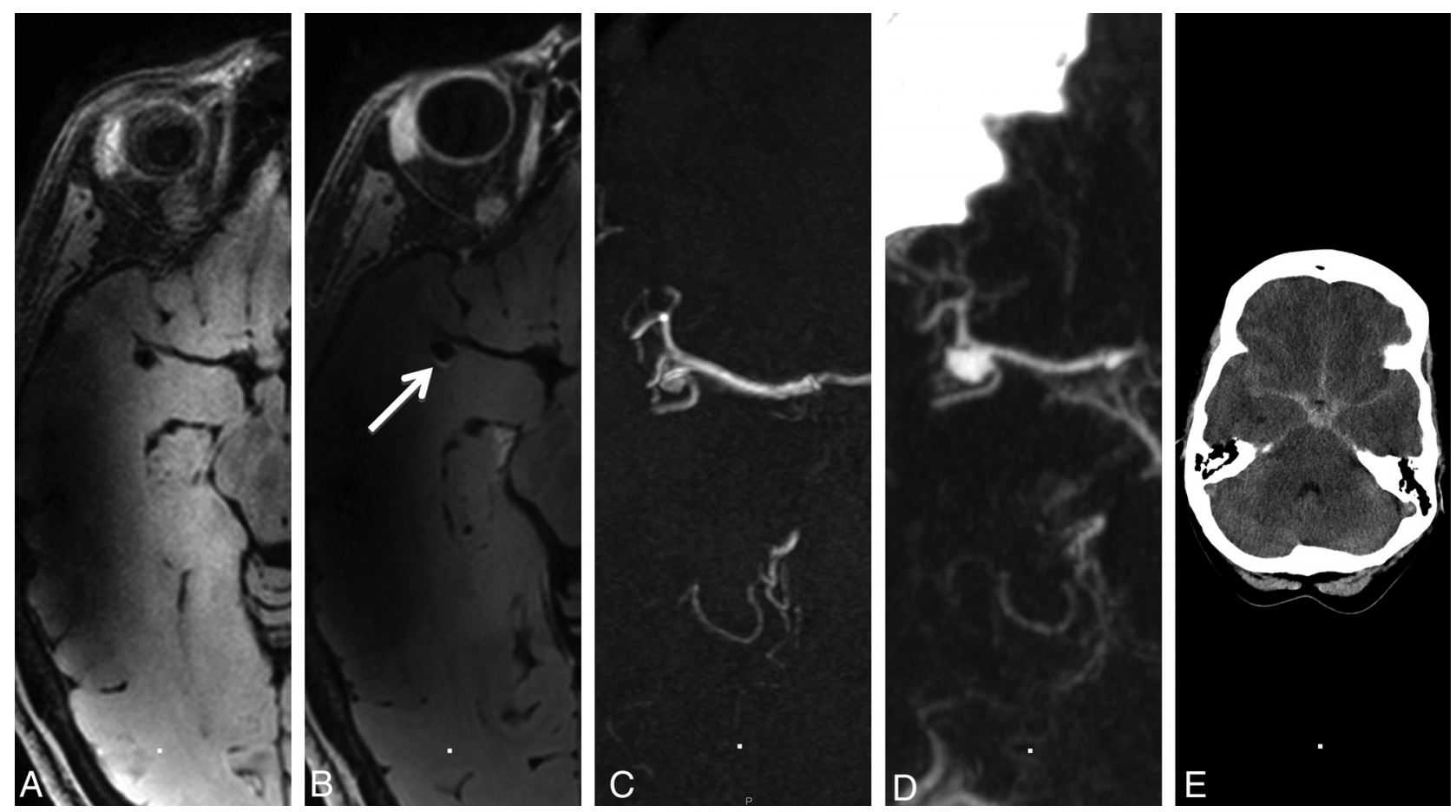

FIG 2. Aneurysm wall imaging of a patient with aneurysm rupture at follow-up. A 47-year-old woman with a right middle cerebral artery aneurysm. A, Aneurysm wall imaging without gadolinium. $B$, Aneurysm wall imaging after administration of gadolinium shows focal posterior enhancement (arrow). C, At baseline, the aneurysm is $4 \mathrm{~mm}$. D, After 31 months, the aneurysm ruptured, with CTA showing aneurysm growth $(7 \mathrm{~mm})$. E, Head CT shows SAH.

12 unstable aneurysms, which were defined as a recently ruptured aneurysm, symptomatic aneurysm, or growing aneurysm. ${ }^{11} \mathrm{~A}$ fifth study found aneurysm wall enhancement in $74 \%$ of unruptured intracranial aneurysms and in all 19 ruptured aneurysms. ${ }^{14}$ The LUMINA study did not include ruptured aneurysms and found wall enhancement in $29 \%$ of 89 small unruptured intracranial aneurysms. ${ }^{12}$

Current risk prediction for aneurysm growth and rupture is based on the earlier subarachnoid hemorrhage, location of the aneurysm, age $>60$ years, population, size of the aneurysm, and shape of the aneurysm (ELAPSS) and PHASES scores, which were designed on the basis of large cohorts of patients with unruptured intracranial aneurysms. ${ }^{4,5}$ In these scores, risk prediction is based on patient and aneurysm characteristics, with aneurysm size, aneurysm location, and demographics being the most important contributing variables. However, because most instances of SAH are caused by rupture of a small aneurysm, novel biomarkers are needed that predict aneurysm instability and will lead to a personalized approach to risk prediction. The results of our study suggest that aneurysm wall imaging with gadolinium administration is such a novel biomarker and therefore an ideal tool for accurately selecting patients who will have aneurysm instability in the short term.

A strength of our study is that it had a longitudinal instead of a cross-sectional design. In addition, all patients had aneurysm wall imaging before and after administration of gadolinium according to strict predefined protocols. We included patients in whom the treating physician had decided on follow-up imaging instead of preventive aneurysm occlusion. Specifically in this group of patients, novel biomarkers are needed to better predict which aneurysms have an increased risk of instability during follow-up. Finally, because gadolinium contrast agents are safe and widely available, it is easy to implement MR imaging with gadolinium-enhanced aneurysm wall imaging in clinical care.

A limitation of our study is the single-center design, which limits generalization of our results to other populations. In addition, baseline imaging was either with $3 \mathrm{~T}$ or $7 \mathrm{~T}$ MR imaging. It remains unclear whether $7 \mathrm{~T}$ is more sensitive to detect aneurysm wall enhancement than 3T MR imaging. Furthermore, follow-up imaging was performed with both CTA and MRA, instead of MRA only. Another limitation of our study is its relatively small sample size. Finally, because all aneurysms with instability during follow-up were $>5 \mathrm{~mm}$, it remains to be investigated whether aneurysm wall enhancement is a predictor of aneurysm instability independent of aneurysm size.

Our study has implications. The results of this study suggest that aneurysm wall MR imaging with gadolinium administration can be used to improve patient selection for preventive neurosurgical or endovascular aneurysm treatment in patients with small, unruptured intracranial aneurysms. However, because of the currently still imprecise results, larger studies are needed to confirm these findings and to investigate whether aneurysm wall enhancement has predictive information of future aneurysm instability in addition to the current predictors.

\section{CONCLUSIONS}

Gadolinium enhancement of the aneurysm wall on MR imaging is associated with an increased risk of aneurysm instability. The ab- 
sence of wall enhancement makes it unlikely that the aneurysm will grow or rupture in the short term.

Disclosures: Mervyn Vergouwen—UNRELATED: Payment for Lectures Including Service on Speakers Bureaus: Cerenovus. * Money paid to the institution.

\section{REFERENCES}

1. Vlak MH, Algra A, Brandenburg R, et al. Prevalence of unruptured intracranial aneurysms, with emphasis on sex, age, comorbidity, country, and time period: a systematic review and meta-analysis. Lancet Neurol 2011;10:626-36 CrossRef Medline

2. Vergouwen MD, Jong-Tjien-Fa AV, Algra A, et al. Time trends in causes of death after aneurysmal subarachnoid hemorrhage: a hospital-based study. Neurology 2016;86:59-63 CrossRef Medline

3. Algra AM, Lindgren A, Vergouwen MD, et al. Procedural clinical complications, case-fatality risks, and risk factors in endovascular and neurosurgical treatment of unruptured intracranial aneurysms: a systematic review and meta-analysis. JAMA Neurol 2018 Dec 28. [Epub ahead of print] CrossRef Medline

4. Greving JP, Wermer MJ, Brown RD Jr, et al. Development of the PHASES score for prediction of risk of rupture of intracranial aneurysms: a pooled analysis of six prospective cohort studies. Lancet Neurol 2014;13:59-66 CrossRef Medline

5. Backes D, Rinkel GJ, Greving JP, et al. ELAPSS score for prediction of risk of growth of unruptured intracranial aneurysms. Neurology 2017;88:1600-06 CrossRef Medline

6. Bender MT, Wendt H, Monarch T, et al. Small aneurysms account for the majority and increasing percentage of aneurysmal subarachnoid hemorrhage: a 25-year, single institution study. Neurosurgery 2018;83:692-99 CrossRef Medline

7. Matouk CC, Mandell DM, Günel M, et al. Vessel wall magnetic resonance imaging identifies the site of rupture in patients with mul- tiple intracranial aneurysms: proof of principle. Neurosurgery 2013; 72:492-96 CrossRef Medline

8. Edjlali M, Gentric JC, Régent-Rodriguez C, et al. Does aneurysmal wall enhancement on vessel wall MRI help to distinguish stable from unstable intracranial aneurysms? Stroke 2014;45:3704-06 CrossRef Medline

9. Nagahata S, Nagahata M, Obara M, et al. Wall enhancement of the intracranial aneurysms revealed by magnetic resonance vessel wall imaging using three-dimensional turbo spin-echo sequence with motion-sensitized driven-equilibrium: a sign of ruptured aneurysm? Clin Neuroradiol 2016;26:277-83 CrossRef Medline

10. Liu P, Qi H, Liu A, et al. Relationship between aneurysm wall enhancement and conventional risk factors in patients with unruptured intracranial aneurysms: a black-blood MRI study. Interv Neuroradiol 2016;22:501-05 CrossRef Medline

11. Hu P, Yang Q, Wang DD, et al. Wall enhancement on high-resolution magnetic resonance imaging may predict an unsteady state of an intracranial saccular aneurysm. Neuroradiology 2016;58:979-85 CrossRef Medline

12. Backes D, Hendrikse J, van der Schaaf I, et al. Determinants of gadolinium-enhancement of the aneurysm wall in unruptured intracranial aneurysms. Neurosurgery 2018;83:719-25 CrossRef Medline

13. Lv N, Tang H, Chen S, et al. Morphological parameters related to aneurysmal wall enhancement in patients with multiple intracranial aneurysms. World Neurosurg 2018;114:e338-43 CrossRef Medline

14. Wang GX, Wen L, Lei S, et al. Wall enhancement ratio and partial wall enhancement on MRI associated with the rupture of intracranial aneurysms. J Neurointerv Surg 2018;10:566-70 CrossRef Medline

15. Kleinloog R, Zwanenburg JJM, Schermers B, et al. Quantification of intracranial aneurysm volume pulsation with 7T MRI. AJNR Am J Neuroradiol 2018;39:713-19 CrossRef Medline

16. Kleinloog R, Korkmaz E, Zwanenburg JJ, et al. Visualization of the aneurysm wall: a 7.0-Tesla magnetic resonance imaging study. Neurosurgery 2014;75:614-22; discussion 622 CrossRef Medline 